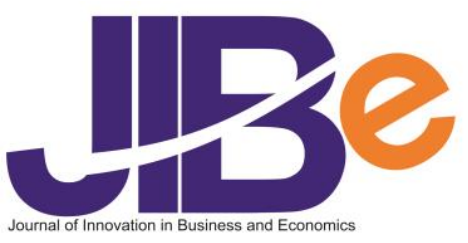

\title{
The games of imitation: AI and a philosophy towards future equilibrium
} Todd J. Barry ${ }^{1}$

\author{
Hudson County Community College, Connecticut, United States
} of America ${ }^{l}$

\begin{abstract}
This brief conceptual article starts with an argument for Artificial Intelligence (AI)'s ability to "think." This outgrowth relates to human's and AI's power over nature, and to AI's increasing power in its humanness, measured by the results of competing with humans and other AI machines in the Turing Test, and economic "game theory." Both, and especially the latter challenge, can be quintessentially human by measuring how one values the self as opposed to society, under varying conditions. Given AI's advancements enabling it to presumably "win" in the most humanness of games, beyond even reaching a universally beneficial "social optimal" outcome, and thus possibly even having more power than humankind, the article argues for an equilibrium of balanced powers in innovation between AI and humans. Therefore, managers, broadly construed, can function as key brokers between government policy makers and innovators as $\mathrm{AI}$ and humans continue to develop further into the future.
\end{abstract}

Keywords: Artificial Intelligence; Game Theory; Turing Test; Nash Equilibrium

\section{Introduction}

According to Mumford (1934), humankind began using tools to develop them as an extension ourselves, to give us more "power" over nature. Today, humankind's environment has expanded to the economic, social, and political spheres. Now, robots, or Artificial Intelligence machines (AI), which are extremely, sophisticatedly programmed computers, can almost "stand on their own." In the 1950s, Alan Turing developed an hypothesis, known as the "imitation game," to judge how far AI had, or would, come. The test of whether AI has fully become human is in-part measured by this imitation game. But, subsequently, humanness and human power can also be assessed by AI playing humans in other games, some known in economics as "game theory." Based on philosophy, would the computer be able to consistently defeat the human, in Turning's test, or in some type other type of "game theory," why or why not, and if it did, would this make it more of a human? In doing so, would this signal that AI has more power over nature than humans, and even over humans ourselves, and how will this affect the societal structure of the future?

This paper contribute to the existing literature by not only pointing out the benefits and flaws of AI, but by suggesting that given the technological and "humanness" advances in AI, a future equilibrium can be reached, contrary to the writings of Jonas (1979). The equilibrium could be called: a balance in the use of tools over nature, a "socially optimal" outcome in game theory, "utilitarianism" in philosophy, as a marginal revenue/cost or cost-benefit analysis in microeconomics, products with new features yet problems (externalities or "dead weight" loss) created under managers' oversight, or as checks and balances in different spheres of life, the social, economic, and political. It is the purpose of this article to show that in all of these cases, humankind's and AI's powerfulness can be equal, with power defined as their ability to "win" in human interaction and thus have the ability to enact change in the different spheres. To start, the paper begins with a discussion of concepts of which show the remarkable advances in the power of AI, the ability to "win" at the Turing Test (or imitation game) and at "game theory" in economics, demonstrating an intellectual power. The paper thus employs a philosophical and hypothetical test methodology.

The Turing Test, or imitation game, can test human versus AI power. The test refers to placing an advanced computer and a human in a room, having them answer questions, from a researcher, all having the ability to lie and deceive, such that the researcher cannot tell which one is the human, and which is the computer. Early tests involved writing down answers for the researcher, because AI's voice system was not fully developed, as it is not completely so today, if one has experience of talking with Siri, or automated telephone calls. A different game, called the "Chinese Room," involves giving computers pages written in

\footnotetext{
${ }^{1}$ E-mail: tjbarry00@yahoo.com
} 
different languages and seeing if one can decipher the meanings. Are not symbols the very essence of human intellect, even including our ability to represent God through speech, or for our symbolic name/s for $\mathrm{Him} / \mathrm{Her}$ ?

A long held debate involves whether or not computers can actually think, or if they are just reading lines off of a piece of paper (input-output), so to speak, such as advanced by Searle (1980). But, if AI computers can form images in their programming from seeing or hearing words, and "be present in the room," unlike "Chance the Gardener" in Kosinski's Being There (1999), your author concludes that AI can indeed think. They can learn, and have forms of emotions and values, or at least they will be able to within a short time span. Even with humans, our degree of being present in the room can vary, from those who are more observant, to those who prefer their own, inner, quiet solitude. Both mental illness and mental intelligence are judged by both: our inner knowledge, the ability to think abstractly and not just in terms of objects and events, but in the sense of what is around us, as Ong (1982) surmised. Although AI's experiences will not be exactly the same as humans (they will not have experienced the concept of birth, or social stages in life in the same way), AI's development will occur so long as humans are able to program them to be aware of their own values, from their use of simultaneously running computer programs, which they will then mend according to personal experiences, leading to intentional, individual purposes.

In this paper, "thinking" means that AI can deviate from their programs to find more efficient means of meeting the goals with which we program them. Learning refers to building on their programs their trial and error, or experience or interaction, to change their memory and storage. Machines have a degree of emotions, when outside actors are conflicting with their goals or purpose, but the emotions will develop from calculations and not be as socially spontaneous, and presumably not be centered on a desire to reproduce, in a Freudian sense. And, AI may have values, which means that they can change their purposes, if we allow them, through algorithmic programming, to so-call "evolve."

Lastly, AI may have common sense, to pick up on social cues and everyday problems without detailed programming. The only difference between humans and AI might be Turning's (1950) "religious objection," that, while they may have "souls," they may not go to heaven, as described in popular Christian culture since the Middle Ages. Do members of all different faiths go the same heaven if they do not want to- do Buddhists go to the heaven that they perceive and wish to go to, and Christians to their heaven, and those who wish to be reincarnated be reincarnated? Perhaps it relates to one's subconscious- will AI develop one? Will AI have its own religion- will it seek "something greater" from humankind, seeing us as its maker? Or, will humans just be the "middlemen" between God in the creation of new beings? These are questions for future papers and theologians.

The Turing Test refers to whether AI can "think"- that is, how AI and humans, given the power to lie and deceive, will try to fool or convince the researcher that they are human. Alan Turing believed that "humanness" was measured by human intelligence. To fool the machine in the Imitation Game, the best way might be to present it with a new poem, art work, analogy, or riddle, none which have been created before, such that it would not be already be programmed into AI's memory. Then, ask it to interpret it using emotions, imaginations, concepts, and ideas, all of which AI is weakest in, particularly the last two.

Under "game theory" in economics, AI and the human could pursue a "dominant" strategy, in which they both would try to trick the human, or they could pursue a "socially optimal" strategy, in which case they would play for a draw. This is similar to how, for example, if Bonnie and Clyde were to be arrested, would they turn on each other, or both admit to committing the crime, thus engendering for themselves a lesser, or greater, penalty, depending on the rules, that is, the options that the police present to them, known as the "prisoner's dilemma." Machines, like Turning said, for the "imitation game" itself, would stand a good chance of winning over $70 \%$ of the time, which should be even greater today. An even deeper question might be to study AI's ability to determine a human from another AI machine.

Game theory, which can take many forms, is also concerned with "fooling" an opponent, but it has societal implications. The theory is explained most simply by looking historically at the soldiers involved in trench warfare in World War I. Over time, soldiers and their enemies became used to staring across enemy lines at each other, and made a mutual decision, communicated via no other process than simple reason, to not fire at each other, thus eliminating being killed. In the Cold War, John von Neumann developed this idea for the Cold War, helping to develop the concept that the United States and Soviet Union would not use nuclear weapons due to mutually assured destruction. John Nash, as depicted in the Academy Award winning movie A Beautiful Mind, applied the idea to business, and since then, it has applied to natural selection in biology to competition versus collaboration in other disciplines.

Consider this additional example, which has been used on European, and some American, t.v. game shows. Two players are given $\$ 1,000$ dollars. They can choose to keep the full $\$ 1,000$, or to share it and earn only $\$ 500$. If one chooses to keep and the other chooses to share, the one who chooses to keep 
wins. If both choose to keep, then they both receive $\$ 0$ dollars, and the world ends in proverbial nuclear war. If both choose to share, they split the money and both share, earning $\$ 500$ dollars each. The first strategy, of keeping, is called the "dominant" strategy, while, to share, we shall call the "socially optimal" strategy. Most of the time, players choose they dominant strategy, unless each side comes to trust each other over time, such as what happened with the United States and the Soviet Union.

\section{Literature Review}

The authors mentioned so far, such as Mumford (1934), and Searle (1980), used historical process tracing as a form of qualitative philosophy as their method, while today, some authors with access to technology are actually performing experimental social tests on AI. Most of the qualitative philosophy on AI continues to argue over whether or not AI can "think" and whether or not it is "rational." This often returns to Godel's arguments, writing in the 1930's, whose philosophies ignited a wave of papers in the 1960s, that humans are more powerfully intellectually than computers because humans do not know something simply since we are programed to, but also because we feel the need to verify it either by exploring nature or our inner selves philosophically (Dietrich, 2020).

In terms of tests, according to Ray (2019), AI called AlphaGo Zero has been able to defeat humans at various complex games by 'using a statistical approach called a 'Nash equilibrium,' which finds strategies that beat or tie [the records of a] mixture of [ranked] players" (Ray, 3). This approach of studying human masters, ironically named, is used to win in sequential games when AI cannot see the moves, or previous moves; this will be addressed. Nisioti (2018) writes about reinforced learning, with rewarding AI for achievements, but notes: "what about the effect learning will have on the behavior of the agent? Won't interaction affect its strategy?" (Nisioti, 8). This would affect public games versus blind games. She writes about percentages in games being replaced by populations in dealing with real life situations. Conitzer (N/A) points out that AI can be programmed using a variable "p," which is common knowledge, and that a random element E can be added to make it "strictly... a mixed strategy" in playing simulation or real-life games (Conitzer, 11).

Many scholars focus on the gains to be made to economic growth via AI, such as Di Vaio et al. (2020), but who note business cultural drift in the presence of AI, or, conversely, the dangers of AI, such as to jobs lost or to liability in physical violence (Geisel, 2018). Katsamakas and Pavlov (2020) write that managers can use AI to speed up and enhance business models, lowers prediction costs, and leverages existing data. Geisel (2018) writes that there are many definitions of what constitutes AI, but that leading scientists (including Bill Gates, Elon Musk, and the late Steven Hawking) sent a letter to an international committee warning that the most advanced AI could be end up being more dangerous than even nuclear weapons, not just as in warfare, such as Barry (2018) writes about. She continues: "Self-aware AI machines will be able to create more machines, and there is always the danger whether they will see the need for human beings at all" (Geisel 2018, 117), thus taking over the political sphere, and not just the economic. Writes Afrouzi, "AI (in political control) would be too powerful to be in a single person or a small groups command" (Afrouzi 2018, 3).

Afrouzi also points out that AI could collude amongst itself to benefit in business, such as through secret agreements between traveling agencies and hotels (Afrouzi 2018). He cites Daniel Dennett (1987) as writing that we can understand AI only to an extent, because we cannot answer the "why" questions of how AI behaves, just as we cannot always understand humans, and that "simulational philosophy" (games or tests) of attempting to understand AI behavior cannot measure "pleasure or pain," or how far "rewards" will go in teaching AI, echoing Nisioti (Afrouzi, 2018).

A number of authors, such as Teichert (2018) point out that philosophy is especially suited towards studying AI, especially in the recent works by John McCarthy (2006), who pointed out the philosophical conditions for doings so, and even agreed with others that AI, to be considered human, should be able to pass job tests. Artificial Intelligence can benefit humans since they can automate (speed up) humans, or can speed up themselves, which are key functions in how AI can assist in business, although businesses are usually short-sighted towards the problems they engender (Teichert 2018). Reichling (1996), however, suggests that aside from using philosophy to study AI, the study of philosophy in American colleges and universities has been in decline. Along this line, few authors lay out a philosophy as to how AI and humans can be balanced, the gap-analysis of which is presented in this article following a series of simulations to test whether or not AI will "play" for a "socially optimal" equilibrium. 


\section{Research Method}

The methodology is philosophical analysis, of simple non-recorded (blind) move or outcome games, simple non-recorded move games with reported outcomes, and both types of recorded and reported games (public) with sequential moves. The epistemological viewpoint is that of traditional philosophy applied to AI, that by assigning to it human characteristics, so-called "anthropomorphism," we can compare AI to humans. An additional philosophy used here is that of a cost-benefit analysis, or marginal revenues equaling marginal costs, which was first put forth in the writings of the economist Alfred Marshall in Great Britain in the 1880's. An equilibrium which is best for society is reached when the total benefits to all "players" is maximized, a concept in economics called "utility," which was elaborated upon slightly earlier than Marshall in the 1830's by British philosophers Jeremy Bentham and John Stuart Mill, through socalled "utilitarianism."

The outcome of the games, and whether or not AI and humans reach an equilibrium, may depend on a number of factors, which likewise affect humans, analyzed in-depth below:

a. Ego: of course, a game in which the outcome is not recorded, which would eliminate "ego," or one where the moves are not recorded, would prevent bias or habits in the future. This scenario is mostly hypothetical, because, as scientists, we want to see the results. For humans, choices would highly depend on whether or not the outcome was reported. Artificial Intelligence would care little if the outcome was reported, being more logical and highly less emotional. Unlike chess players, AI would have less ego, which is colloquially defined here as trying to impress others, or gaining a sense of superiority. In an experiment run by your author in his economics class, male participants were concerned with "showing up" their opponents, and impressing the class, therefore choosing the dominant strategy. Artificial Intelligence might develop an "ego" in the sense that it knows it can "win" in the future, and its programmers certainly would have an "ego."

b. The risk: consider a few dollars versus a nuclear war, in which case both parties might take more risk in the former than the latter, pursuing a dominant strategy. Thus, AI can be programmed with values such that it knows if $\mathrm{A}>\mathrm{B}>\mathrm{C}$. It might be able to change its values through learning, but conflicts in such rankings could cause the machine to shut-down.

c. Would AI behave differently knowing it is playing a man or woman, young or old? Women have typically been theorized to more likely "share," confirmed by several studies. In terms of age, this depends if AI has access to historical data, being "young," or "old," with wisdom being the ability to put community ahead of itself (this is in-part why God created humans, say Biblical scholars)again, would AI view humans as part of its community, just AI, or neither? This may make game theory the ultimate human-as-possible test. Humans have a connectivity which Christian theologians might call "the Holy Spirit," while AI computers have been found communicating with each other in the own language, and were shut down.

d. Would the machine have care regarding harming humans, or harming AI, it if were playing an AI machine instead? It would understand the value of life, artificial or human, having been programmed as such, but would we care about the value of AI, as we could simply build another one if we destroyed it, such as with a nuclear weapon? Each AI would learn and thus act separately. Would the computer play the game differently if it were playing another AI, having developed a sense of comradery? That is, would it value other AI greater than it would value humans, and be more prone to choose the "societal value" option? it perchance is likely.

\section{Result and Discussion}

We see from these examples that even the most simple of games can become complex due to such human considerations. Consequently, in a completely non-recorded nor reported game, AI would be logical to choose the "socially optimal" outcome. However, in a reported move single game, or human versus machine, then AI would decide mostly by chance, 50-50\%, in order to keep its record as a vague, to fool its opponents, the programming of which has already been discussed, with an error term. The game would be reduced to luck. This is, of course, assuming that the AI machine has equally considered the individual 
gains for attempting to "win," with societal gains and losses, as we have initially programmed it, with these values.

In a repeated game, the AI machine would probably follow the popular "tit for tat" strategy," responding to humans' moves based on their last one, which is similar to a so-called "carrot and stick" approach. Still, AI may know all of its opponents' moves from past games, but humans as well would know all of the AI machine's decisions from the past. In a repeated game, most economic "games" might end up being a draw, at the socially optimal level, over time, because the two competing parties develop trust between them. We would trust AI, and would AI trust us. The first concept is one that is reshaping the world of innovation- would we trust AI to perform heart surgery on us, to run our bank, or to sell us a car? In certain professions, one would think that at some level, a degree of human interaction would be necessary. Can computers be prejudiced, and treat different demographic groups differently, to predict our behavior, whether from selling mortgages and loans, to playing economic "games"?

More importantly, in a long-term game, even if $\mathrm{AI}$ is kept blind from knowing its opponent, the AI machine may be able to tell the type of person it is playing against, in the terms from Part III, adapting as such, and therefore would be likely to defeat humans. This is reportedly what happened in the famous chess series between Gary Kasparov and IBM's Deep Blue: the machine was programmed, Kasparov latter claimed, to defeat himself personally. The machine, though, might run into problems facing somewhat like Magnus Carlsen, who makes more unpredictable, but equally talented, moves. This is the old debate in chess: does one play the board, or the player? This would amount to AI not only counting the cards, as in poker, but predicting the cards, which we know from Part II are systems that tech managers are now developing.

According to the above analysis, AI should be currently, or in the future, defeat humans at games most of the time, but it depends on the type of game, and over time, and with conditions, the "socially optimal" equilibrium can be reached. Nevertheless, does "winning" in game theory make AI more human, or more powerful than humans? An "economic" game with AI would amount to whether or not AI understands humans more than humans understand AI, a test of both intellectual and social prowess, which could have larger applications. Some might argue that it would simply mean that AI has different strengths and weaknesses compared to humans. Namely, these are its ability to predict, to perceive risk, and to make logical decisions under pressure. This is, of course, defining humans by our qualities, and our ability to "do," as so-called "homo fabers."

A greater question perchance is why do we need to know who will "win"? Do we fear AI, because it may be smarter than us, or, in the opposite case as with automated telephone calls, we do not believe it is as smart as us? Perhaps, we question that it will be even more human than we are? Or, do we not trust it, because who do not know who the programmer was, or from what country, and what values they may be programmed it with? Or, maybe we fear it because we do not understand it, and we do not know how to behave around it, if we should treat it as a human being, or if all AI machines are the same, in a somewhat prejudiced view. Is this why we give AI names like Siri or Alexa, such that names are not just "dog tags," as Ong (1982) agreed, but rather convey a degree specialness in identity, to belong and be referenced to in spoken word as part of our society? Most likely, it is because we like to consider that, just as in the Turing Test, the humans involved have more power over humanlike AI. Conversely, does "winning" make AI think that it is more human, as in Cooley's (1902) psychological "looking-glass self"? In science fiction, it is often a primary goal of technology to try to become more human, and if we program AI to think in terms of humanness, then perhaps "winning" will in-fact shape its self-view.

\section{Conclusions, suggestions and limitations}

Managers of business are on the "front-line" of working to produce AI, for good, or for bad, overseeing in their limited purview the economic power over of humans and AI over society and culture. Yes, they, along with political leaders, will have to deal with the changes in job types and in retraining efforts, but more in-line with your author's paper, they will have to keep a watchful eye over what technology is being created and whether or not it is too powerful. They can provide responses to lawmakers on AI's progression and make sure that lawmakers' priorities are best reached, not just consumers', in terms 
of products and practices. Barry \& Aho (2016) suggested that the economic sphere of life could be best run by AI and that politics would be best run by humans; perhaps, though, they both could use human and AI "checks and balances," along the lines of the French philosopher Montesquieu's writings that were used for the early American Republic (see Figure 1). Here, each of society, economics, and politics is checked in some fashion by both humans and AI. Therefore, MPai (marginal power of AI) $=\mathrm{MPh}$ (marginal power of humans). Humankind need not surrender powerful to an authority greater than itself, nor limit its abilities to conquer nature, and therefore reach a power equilibrium.

To conclude, the culmination over thousands of years since the first stone tools and the first cave paintings in southern France and the Caucuses have resulted in mankind creating and using Artificial Intelligence (AI). In essence, this takes away that very power and seeds it over to something possibly even more powerful than ourselves, even in the case of "cyborgs," human and AI collaboration. Did humans create AI so it can do all jobs for us to improve our economic efficiency? Or, is it is the limit of human achievement that we are trying to proceed with for the sake of knowledge, advancement, and creativity alone? As this paper has shown, this can be seen in the "imitation game," where humans and computer attempt to deceive or identify each other, but do we run this test because we do not want AI to merely imitate us, but to become us, which may be some human's ultimate goal?

With AI being able to "stand as its own," as Mumford (1934) predicted, this power may or may not give us more time for leisure, either in the Greek sense, meaning the time to pursue deeper thoughts and conceive of even greater innovative creations, or, in the plebian sense, to seek the thrills of life. For the former, Mumford calls "art" the "product of being free from the constraints of the natural world." For the later, in terms of microeconomics, the "income effect" versus the "substitution effect" come into mind in our workplaces. The choice refers to whether one prefers more income from more work, or more leisure with the same, or possibly lesser, amounts of income, such that workers may prefer one to the other. Artificial Intelligence, then, hopefully gives humans this economic option.

Machines (AI) today might positively use game theory to function as a "team," such as drones putting out forest fires, destroying an enemy outpost, or preventing groups of self-driving cars from colliding, giving them power over nature. This is leading to research on more innovative "team" games.

Human beings, though, have yet to learn the full potential of AI. As Mumford (1934) wrote about presciently so long ago, we should "assimilate" technology, to find out its best uses, and use it as a natural part of our culture, as we have been accustomed to viewing lines of telephone poles, not see in certain rural areas. Today, assimilation might mean making AI conform to our own values rather the ones they will develop. And, unlike others who have written that there is no "equilibrium" with technology (Jonas 1979, 35), humans conceivably could strive for "equilibrium" in the different spheres of life, the so-called "socially optimal" strategy in game theory, where human power stays equal to AI's, or at least our application of AI does (see Figure 1).

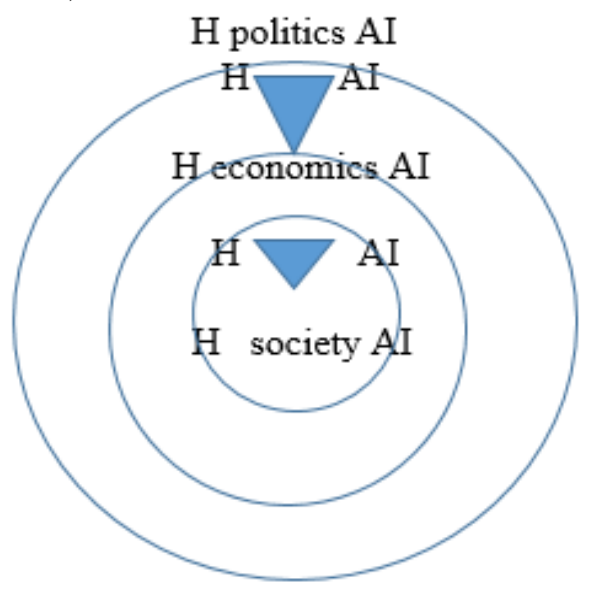

Figure 1. Checks and Balances: This figure depicts triangular checks and balances amongst humans $(\mathrm{H})$ and AI in different spheres of power. The triangles represent points of oversight.

Whether or not this will mean humans physically integrating ourselves with AI remain questionable, as the two could remain separate (see the divisions between humans and AI in Figure 1). While such a collaboration with $\mathrm{AI}$ is a frequent appeal, a corresponding division between AI and human tasks, of check and balances in the spheres of the future society, might too be in order going ahead (see the 
layers and triangles of checks in Figure 1). Katsamakas and Pavlov write that managers must ask themselves: "Do the 'AI feedback loops' (the long-term effects) work for our company? Or (do) they work against our company?" (Katsamakas and Pavlov 2020). Managers, especially those with legal training, and broadly interpreted, might turn out to be the key middle agents between government policies and further innovation, helping to decide what future technology innovations are created or are even legally approved, to keep AI power in equilibrium with humans, or to at least keep lawmakers abreast. Development is by itself intended for the future, for sustainability, but it might be heedful to take caution with AI, since it leaves our power relationship with nature uncertain, and which actors, God- or human-created, will "win."

\section{Acknowledgement}

This paper was inspired by the 2020 St. John's College Summer Teachers Institute- thank you to all of the leaders and participants.

\section{References}

Afrouzi, A.E. (2018). "The Dawn of AI Philosophy.” Blog of the APA. Retrieved December 18, 2020, from https://blog.apaonline.org/2018/11/20/the-dawn-of-ai-philosophy.

Barry, T.J. \& Aho, M. (2016). "Technological Unemployment and Socio-Economic Development: Historical Perspectives and the Future." Communications in Applied Sciences. Dec. 2016.

Barry, T.J. (2018). "Human vs. Robot Decision Making on the Battlefield: War and Rational Choice Theory." Philosophy for Business (electronic journal), Issue 84, June 23, 2018. https://isfp.co.uk/businesspathways/.

Conitzer, V. (N/A). “CPS 270: Artificial Intelligence: Game Theory.” Duke University. Retrieved August 3, 2020. Available at: https://www2.cs.duke.edu/courses/fall08/cps270/cps270_game_theory.pfd.

Cooley, C. H. (1902). Human Nature and the Social Order. New York: Scribner.

Dennett, D.C. (1987). The Intentional Stance. Cambridge, MA: Bradford.

Di Vaio, A., Palladino, R., Hassan, R., \& Escobar, O. (2020). “Artificial intelligence and business models in the sustainable development goals perspective: A systematic literature review." Journal of Business Research, 121(Dec. 2020), pg. 283-314. DOI: 10.1016/j.jbusres.2020.08.019

Dietrich, Eric. (2020). “Godelian Arguments Against AI.” PhilPapers. Retrieved December 18, 2020, from https://philpapers.org/browse/godelian-arguments-against-ai.

Geisel, Ann. (2018). "The Current And Future Impact Of Artificial Intelligence On Business." International Journal of Scientific \& Technology Research, 7(5), pg. 116-122.

Katsamakas, E. \& Pavlov, O. (2020). "AI and Business Model Innovation: Leverage the AI Feedback Loops.” Journal of Business Models, 8(2), pg. 22-30. https://doi.org/10.5278/ojs.jbm.v8i2.3532

Jonas, H. (1979). "Toward a Philosophy of Technology." Hastings Center Report, 9(1): pg. $34-43$. https://doi.org/10.2307/3561700.

Kosinski, J. (1999). Being There. Reprint Edition. New York: Grove Press.

McCarthy, J. (2006). "The Philosophy of AI and the AI of Philosophy." Stanford University. Retrieved December 18, 2020, from http://www-formal.stanford.edu/jmc/.

Mumford, L. (1934). Technics and Civilization. New York: Harcourt.

Nisioti, E. (2018). "In need of evolution: game theory and AI." Free Code Camp. Retrieved August 3, 2020. Available at: https://www.freecodecamp.org/news/game-theory-and-ai- where-it-allstarted-and-where-it-should-all-stop-82f7bd53a3b4/.

Ong, W. J. (1982). Orality and Literacy: The Technologizing of the Word. London: Routledge.

Ray, T. (2019). "Google's AI surfs the "gamescape" to conquer game theory." ZDNet. Retrieved August 3, 2020. Available at: https://www.zdnet.com/article/googles-ai-surfs-the- gamescape-toconquer-game-theory/.

Reichling, M.J. (1996). “On the Question of Method in Philosophical Research” Philosophy of Music Education Review, 4(2), pg. 117-127.

Searle, J. (1980). "Minds, brains, and programs." Behavioral and Brain Sciences 3: pg. 417-457. https://doi.org/10.1017/S0140525X00005756.

Teichert, Leonhard. (2018). "The Impact of AI on the Future of Work: A Philosophical Approach (Part I). The Hive. Retrieved December 18, 2020, from https://medium.com/ hivedata/the-impact-of-ai-onthe-future-of-work-a-philosophical-approach-part-i-e5aff3bb0a30.

Turning, A. (1950). "Computing Machinery and Intelligence." Mind 59: pg. 433-460. https://doi.org/10.1093/mind/LIX.236.433. 
